\title{
iPSC modeling of severe aplastic anemia reveals impaired differentiation and telomere shortening in blood progenitors
}

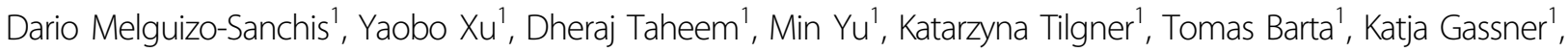 \\ George Anyfantis ${ }^{1}$, Tengfei Wan², Ramu Elango ${ }^{3}$, Sameer Alharthi ${ }^{3}$, Ashraf A. El-Harouni ${ }^{3}$, Stefan Przyborski ${ }^{4}$, \\ Soheir Adam ${ }^{5,6}$, Gabriele Saretzki ${ }^{2}$, Sujith Samarasinghe ${ }^{7}$, Lyle Armstrong ${ }^{1}$ and Majlinda Lako ${ }^{1}$
}

\begin{abstract}
Aplastic Anemia (AA) is a bone marrow failure (BMF) disorder, resulting in bone marrow hypocellularity and peripheral pancytopenia. Severe aplastic anemia (SAA) is a subset of AA defined by a more severe phenotype. Although the immunological nature of SAA pathogenesis is widely accepted, there is an increasing recognition of the role of dysfunctional hematopoietic stem cells in the disease phenotype. While pediatric SAA can be attributable to genetic causes, evidence is evolving on previously unrecognized genetic etiologies in a proportion of adults with SAA. Thus, there is an urgent need to better understand the pathophysiology of SAA, which will help to inform the course of disease progression and treatment options. We have derived induced pluripotent stem cell (iPSC) from three unaffected controls and three SAA patients and have shown that this in vitro model mimics two key features of the disease: (1) the failure to maintain telomere length during the reprogramming process and hematopoietic differentiation resulting in SAA-iPSC and iPSC-derived-hematopoietic progenitors with shorter telomeres than controls; (2) the impaired ability of SAA-iPSC-derived hematopoietic progenitors to give rise to erythroid and myeloid cells. While apoptosis and DNA damage response to replicative stress is similar between the control and SAA-iPSC-derivedhematopoietic progenitors, the latter show impaired proliferation which was not restored by eltrombopag, a drug which has been shown to restore hematopoiesis in SAA patients. Together, our data highlight the utility of patient specific IPSC in providing a disease model for SAA and predicting patient responses to various treatment modalities.
\end{abstract}

\section{Introduction}

Aplastic Anemia (AA) is a rare and serious bone marrow disorder associated with hypocellular bone marrow and peripheral pancytopenia. Severe AA (SAA) is a subtype of the disease characterized by very low bone marrow cellularity of less than $25 \%$, with significant morbidity and mortality ${ }^{1}$. AA occurs with peak incidences at the two extremes of life, in patients between the age of 10 and 25, and patients aged $>60$ years. Children with AA are more

\footnotetext{
Correspondence: Majlinda Lako (majlinda.lako@ncl.ac.uk)

${ }^{1}$ Institute of Genetic Medicine, Newcastle University, Newcastle, UK

${ }^{2}$ The Ageing Biology Centre. Institute for Cell and Molecular Biosciences, Newcastle University, Newcastle, UK

Full list of author information is available at the end of the article Edited by $Y$. Shi
}

often treated with hematopoietic stem cell transplantation (HSCT) while adults are treated with either immunosuppressive therapy using anti-thymocyte globulin (ATG) and Cyclosporine or HSCT, if a matched donor is available $^{2}$. Currently, $70-80 \%$ of cases are classified as idiopathic because their etiology is unknown. The remainder (15-20\%) consists of constitutional bone marrow failure syndromes with the most common being Fanconi anemia (FA) followed by the telomeropathies such as dyskeratosis congenital (DC).

There are currently two proposed models of pathogenesis in idiopathic AA that could explain the characteristic marrow hypocellularity observed in this disorder. In model 1 , an underlying abnormality of the hematopoietic stem 
cells (HSCs) may result in a predisposition to stem cell damage, as well as qualitative or quantitative defects of HSC production. In model 2, a deregulated immune response targets a normal HSC compartment. Strong evidence for an immune component to the pathogenesis of AA comes from the success of the immunosuppressive therapies in treating AA and associated clinical features, including aberrations in immune cell number, phenotype and function ${ }^{2}$. Evidence for an underlying stem cell/progenitor defect is derived from the observations of reduced hematopoietic progenitor cell numbers both at presentation and following successful therapy with $\mathrm{ATG}^{3,4}$, enhanced apoptosis of HSCs, upregulation of genes involved in cell death in hematopoietic progenitors obtained from AA patients $\mathrm{s}^{5-7}$ and mutations in genes such as $P R F 1^{8}, M P L^{9}, N S B 1^{10}, S B S D^{11}, T E R T, T E R C$, and $T I F 2^{12}$ as well as polymorphisms in the IFN gamma $^{13}$ found in a small number of patients.

Acquisition of cells from the bone marrow of AA patients is complicated by the paucity of hematopoietic stem/progenitor cells in these patients and the lack of mouse models which recapitulate the entire spectrum of $\mathrm{AA}^{14-16}$. Here we describe the generation of an iPSCderived disease model for SAA consisting of iPSC lines derived from 1 pediatric and 2 young adult cases and 3 unaffected controls. These iPSC lines were differentiated to hematopoietic lineages and although no significant differences were observed in the ability to give rise to hematopoietic progenitors between control and SAAiPSC, the later, showed a reduced potential to generate erythroid and myeloid cells, impaired proliferation and shorter telomeres when compared to unaffected controls.

\section{Results}

Generation of human iPSC from control and SAA patient fibroblasts

To generate an in vitro disease model for SAA, dermal fibroblasts cells from 1 pediatric and 2 young adult cases of idiopathic SAA, showing severe and very severe clinical manifestation and no clinical stigmata of constitutional AA as well as three unaffected controls (indicated as WT throughout the manuscript) were transduced with the non-integrating RNA-derived Sendai virus $(\mathrm{SeV})$ vector including the four Yamanaka reprogramming factors (OCT4, SOX2, KLF4, c-MYC) as previously reported ${ }^{17}$ (Table 1). SAA fibroblasts showed slightly reduced reprogramming efficiencies compared to unaffected controls (Table 1). No residual presence of the $\mathrm{SeV}$ transgenes was detected in any of the lines generated indicating that SAA-iPSC and control lines successfully maintained stable regulation of the endogenous pluripotent gene expression activated during reprogramming after the loss of $\mathrm{SeV}$ transgene expression (Supplementary Fig. 1). Cytogenetic analysis carried out by single nucleotide polymorphisms (SNP) array showed that the reprogramming process/prolonged culture of the iPSC lines did not induce any detectable genomic abnormalities with the exception of SAA1-iPSC in which two loss of heterozygosity events were identified (Supplementary Table 1). SAA-iPSC colonies displayed characteristic human ESClike morphology and expression of pluripotent markers (Fig. 1a, Supplementary Fig. 2a and b). Similarly, in vivo assessment of pluripotency revealed that SAA-iPSC lines induced formation of teratomae containing cells belonging to all three germ layers (Fig. 1b, Supplementary Fig. 2c). These data indicate that SAA-iPSC and controliPSC lines displayed characteristic features of fullyreprogrammed cells.

\section{Reduced colony-forming potential of SAA iPSC-derived hematopoietic progenitors}

To investigate the hematopoietic differentiation potential of the SAA-iPSC lines, all patient specific and control iPSC were differentiated using a method previously described by Olivier et al. ${ }^{18}$. Early stages of mesoderm induction from iPSC cultures were monitored on day 3 of

Table 1 Table providing detailed information regarding SAA patients used in this study and reprogramming efficiencies obtained for each control and patient

\begin{tabular}{|c|c|c|c|c|}
\hline Patient ID & Age (years) & Gender & Phenotype & Reprogramming efficiency \\
\hline WT1 & Newborn & Male & Heathy & $0.21 \%$ \\
\hline WT2 & 51 & Male & Healthy & $0.29 \%$ \\
\hline WT3 & 37 & Female & Healthy & $0.14 \%$ \\
\hline SAA1 & 16 & Male & Severe AA Responded to immunosuppressors & $0.11 \%$ \\
\hline SAA2 & 24 & Male & $\begin{array}{l}\text { Very Severe AA Responded to immunosuppressors Developed PNH and } \\
\text { relapsed Failed with second course of horse ATG Successful matched } \\
\text { unrelated HSCT }\end{array}$ & $0.10 \%$ \\
\hline SAA3 & 10 & Female & Very Severe AA and autism Successful matched unrelated HSCT & $0.11 \%$ \\
\hline
\end{tabular}


differentiation by expression of KDR (FLK1) ${ }^{19}$. Generation of the first hematopoietic progenitors was detected at day 6 using the $\mathrm{CD} 43$ pan-hematopoietic marker ${ }^{20,21}$. The emergence of hematopoietic progenitors $(\mathrm{CD} 43+)$ and the subtypes of hematopoietic progenitors including megakaryocyte progenitors (CD41a+CD235a-), erythroid progenitors (CD41a-CD235a+), megakaryocyte/ erythroid progenitors $(\mathrm{CD} 41 \mathrm{a}+\mathrm{CD} 235 \mathrm{a}+)$ and myeloid progenitors (CD41a-CD235a-) was assessed by flow cytometric analysis throughout the differentiation time course $^{20}$ (Fig. 2a). To identify the sources of variation that could affect the ability to generate hematopoietic progenitors, different variables such as differentiation experiment, passage number, clonal and donor cell origin (genetic background) were compared using the controliPSC lines by flow cytometric analysis ${ }^{22}$ (Supplementary Fig. 3a). None of these parameters showed a statistically significant difference in the percentage of CD43 positive cells at day 12 (Supplementary Fig. 3b-d). Hence, one clone from each patient and control was used throughout this study. To enable comparison of data from each patient against all three controls, the latter were pooled together, averaged and shown as WT throughout the manuscript.

Analysis of mesodermal induction in SAA-iPSC lines at day 3 revealed that patient cell lines showed similar frequencies of $\mathrm{KDR}+$ compared to control-iPSC lines (Fig. 2b). No significant reduction in the potential to generate $\mathrm{CD} 43+$ hematopoietic progenitors was observed in any of the SAA-iPSC cell lines compared to unaffected controls at days 6 and 12 (Figs. 2c and 3b); however one of the SAA patient iPSC (SAA1) showed a statistically significant reduction in the potential to generate erythroid progenitors (CD43+CD41a-CD235a+) (Fig. 3b).

To assess the colony-forming potential of the SAA-iPSC cell lines, we performed colony forming unit (CFU) assay at day 12 by culturing iPSC-derived-hematopoietic progenitors in methylcellulose-derived media enriched with recombinant cytokines which promote the differentiation into committed erythroid progenitors (CFU-E and BFUE), and myeloid lineage progenitors (CFU-G, CFU-M, CFU-GM, and CFU-GEMM) (Fig. 2a). All three SAAiPSC-derived hematopoietic progenitors showed a significant reduction in the total number of CFUs indicating
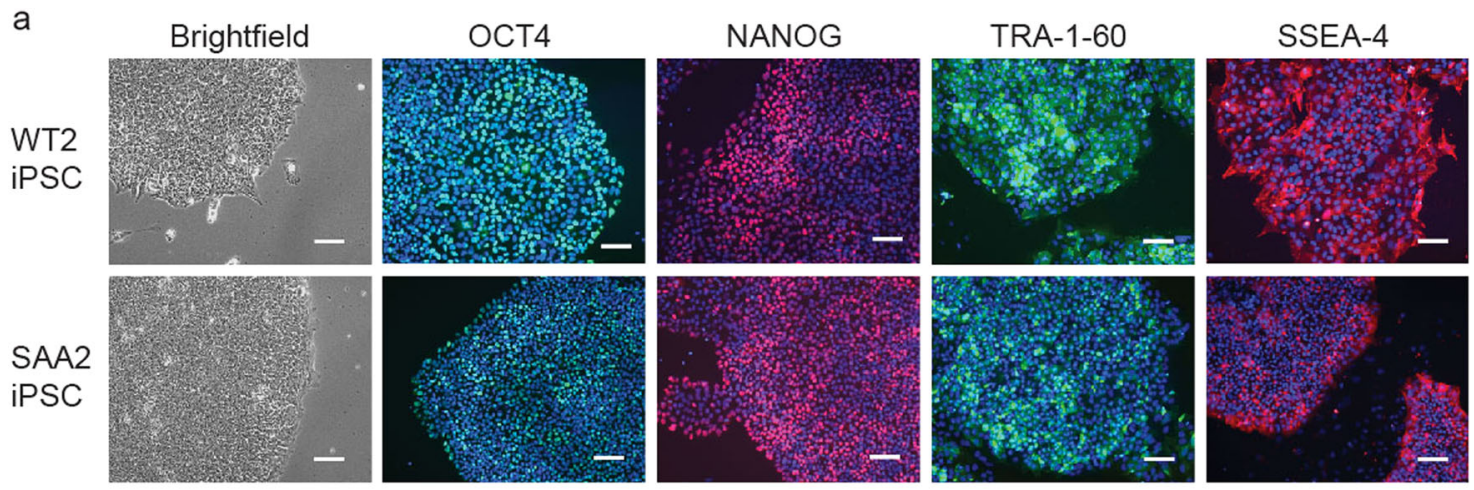

b
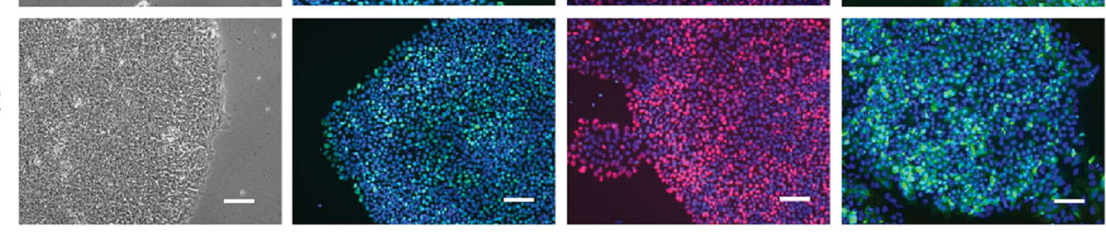

Overall

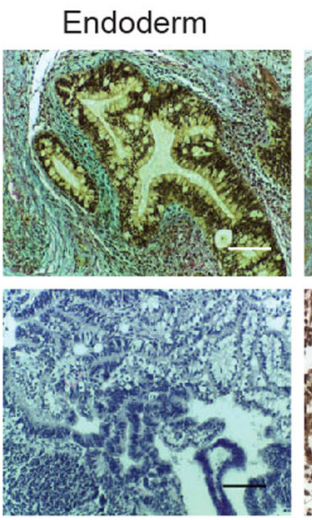

Mesoderm

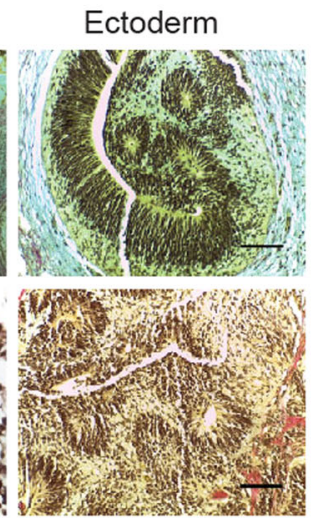

Fig. 1 SAA-iPSC lines display in vitro hallmarks of pluripotency. a Brightfield images of control and SAA-iPSC colonies displaying typical ESC-like morphology and staining of control and SAA-iPSC colonies with pluripotency markers. DAPI staining is shown in blue. Scale bars, $100 \mu \mathrm{m}$; b Histological analysis of representative teratomae generated for control and SAA-iPSC lines displaying trilineage differentiation. Scale bars, overall $500 \mu \mathrm{m}$, ectoderm $100 \mu \mathrm{m}$, mesoderm $200 \mu \mathrm{m}$, ectoderm $100 \mu \mathrm{m}$ 


\section{a}

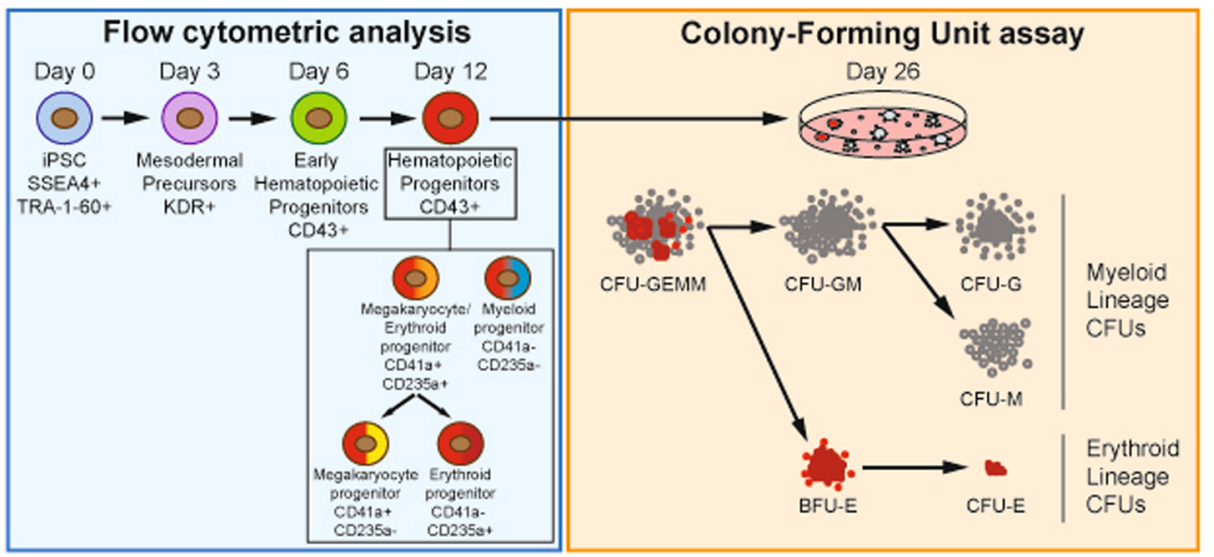

b

Day 3

KDR+
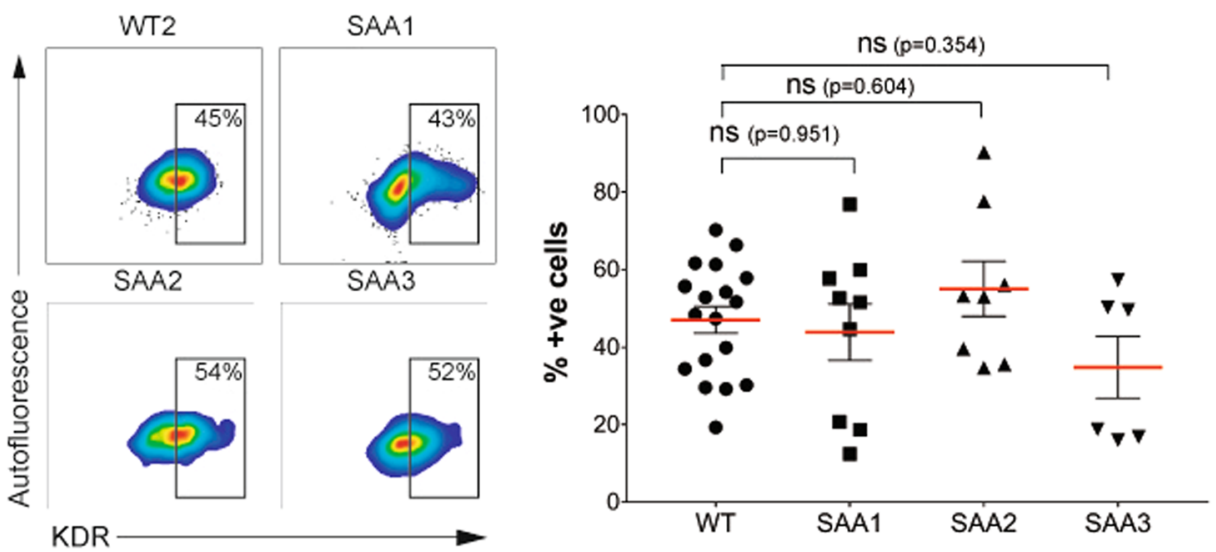

C

Day 6

CD43+
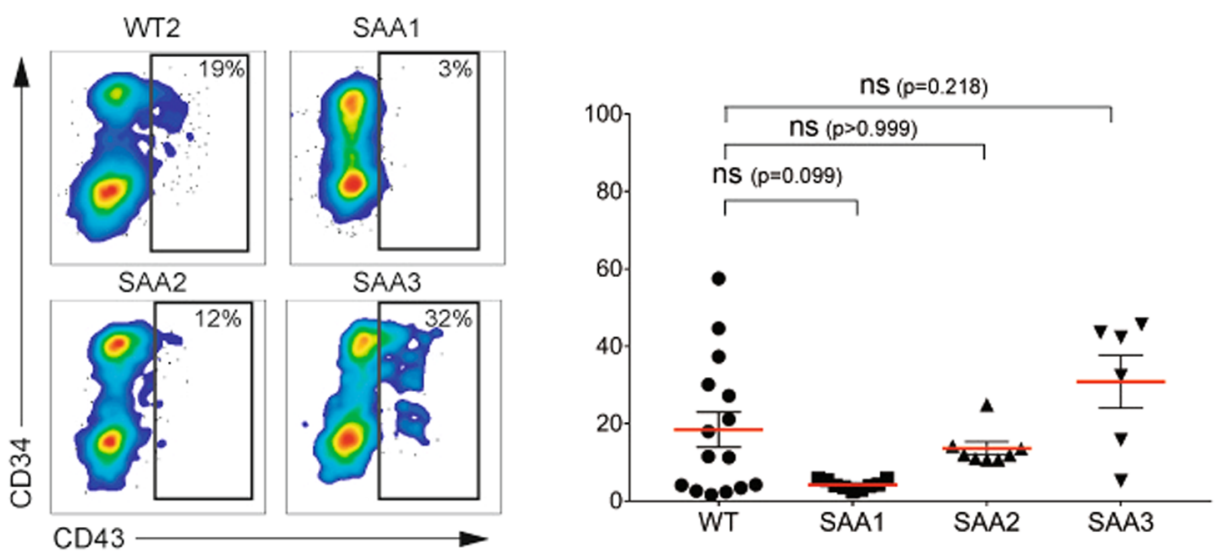

Fig. 2 SAA-iPSC differentiation into mesodermal and hematopoietic progenitors. a Schematic representation of the experimental design used to analyze the WT and SAA-iPSC hematopoietic differentiation capacity; $\mathbf{b}$ Representative images of flow cytometric analysis and scatter dot plot representation of KDR expression in WT and SAA cell lines on day 3. c Representative images of flow cytometric analysis and scatter dot plot representation of CD43 expression in differentiating WT and SAA cell lines on day 6. b One-way ANOVA with Dunnett's multiple comparison test was used for statistical comparison between WT and SAA cell lines. c Kruskal-Wallis with Dunn's multiple comparison test was used for statistical comparison between WT and SAA cell lines. b, c Data is presented as mean of at least 3 independent experiments \pm S.E.M. Data for all control cell lines is averaged in one group (WT) 


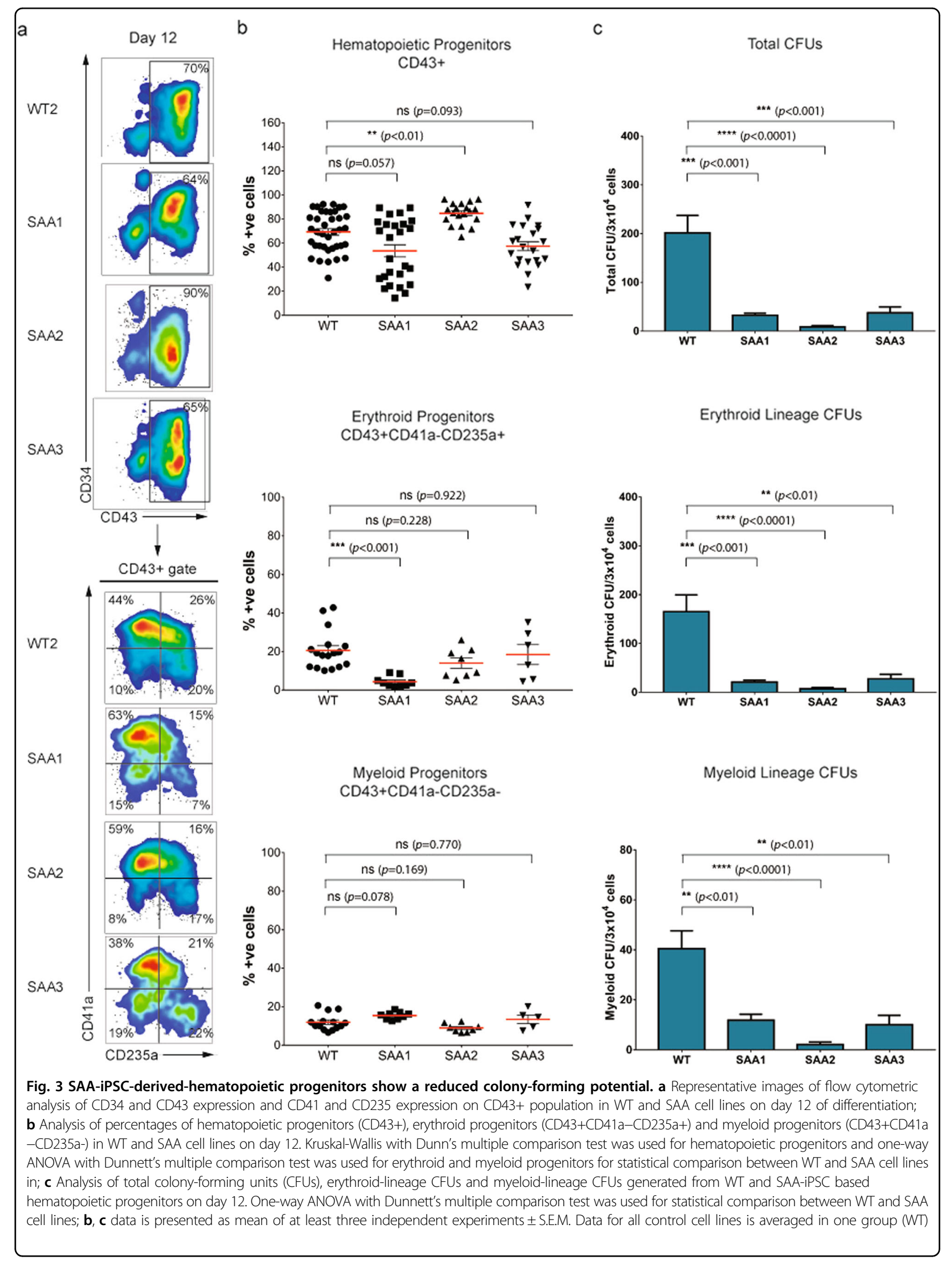


an impaired hematopoietic colony-forming capacity, including both erythroid and myeloid colony potential (Fig. 3c). Despite reduced hematopoietic colony number in SAA, no differences in types and ratios of colonies formed were observed (data not shown). Similar data were obtained at day 16 of differentiation (Supplementary Fig. 4), suggesting that the impaired hematopoietic colony forming ability was not due to a delay in differentiation process.

Telomerase-independent impaired telomere elongation in SAA-iPSC cell lines and enhanced telomere attrition during hematopoietic differentiation

Excessive telomere attrition in highly proliferative cells such as hematopoietic stem and progenitor cells can lead to bone marrow failure ${ }^{23}$. Although the SAA patients used in this study did not display typical dyskeratosis congenita phenotype or mutations in DKC1, TINF2, TERT or TERC genes by exome sequencing analysis (Supplementary Tables 2 and 3), we decided to investigate the telomere dynamics in our SAA-iPSC model since it has been reported that one third of acquired AA patients present short telomeres in leukocytes ${ }^{24-26}$. Control-iPSC showed longer telomeres than parental fibroblasts due to telomere elongation during reprogramming, corroborating published reports ${ }^{27}$. No increases in telomere length were observed during the reprogramming of SAA fibroblasts (Fig. 4a). Furthermore, one of the patients (SAA3) showed continued telomere shortening during the reprogramming process (Fig. 4a), resulting in iPSC with telomeres a

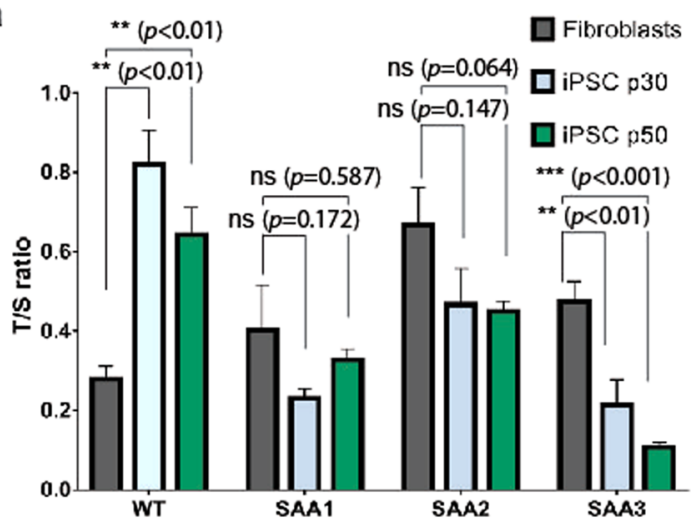

C

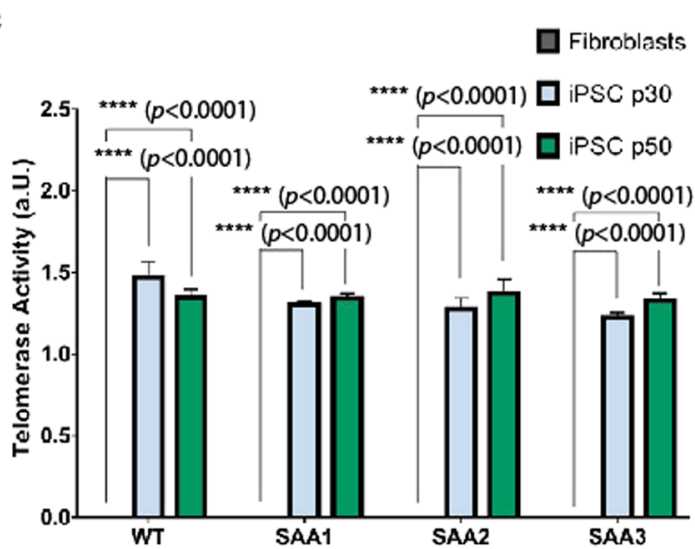

b

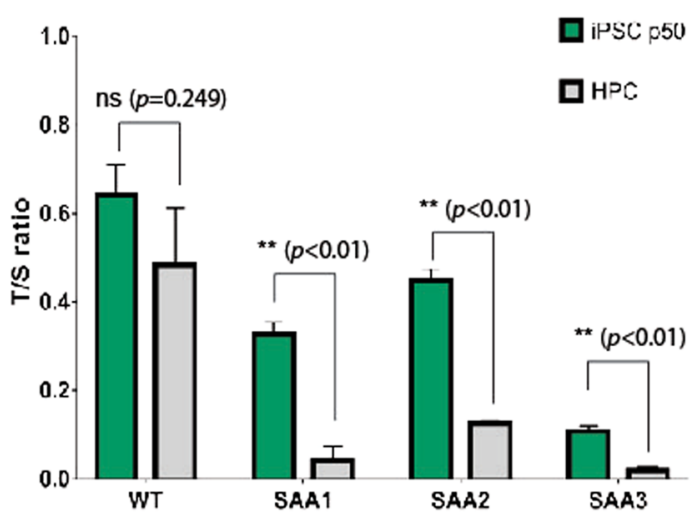

d

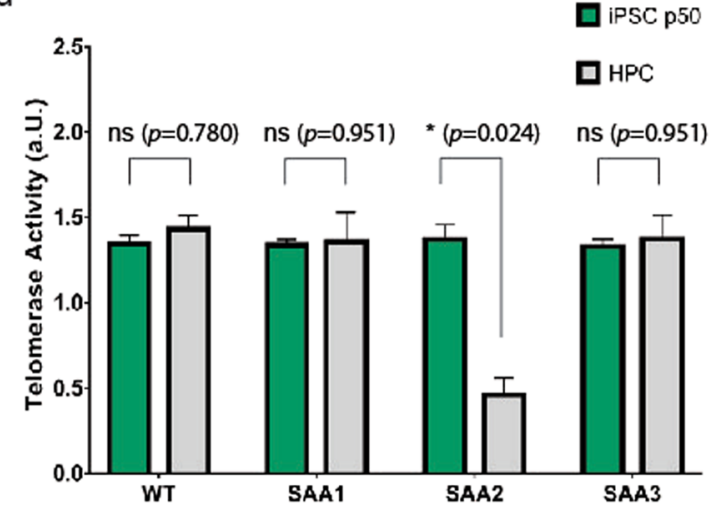

Fig. 4 SAA-iPSC exhibit deficient telomere elongation during reprogramming and telomere shortening upon hematopoietic differentiation. a Analysis of telomere length in parental fibroblasts (dark grey bars) and iPSC passage 30 (light blue bars) and 50 (green bars) in WT controls and SAA cell lines. One-way ANOVA with Tukey's multiple comparison test was used for statistical comparison between fibroblasts and iPSC passage 30 and passage $50\left({ }^{*} p<0.05\right)$; b Analysis of telomere length in IPSC passage 50 (green bars) and iPSC-based hematopoietic progenitors (HPC) differentiated from iPSC passage 50 (light grey bars) in WT controls and SAA cell lines. Multiple t-test using Holm-Sidak method was used for statistical comparison between iPSC passage 50 and HPC; c Analysis of telomerase activity in parental fibroblasts (dark grey bars) and iPSC at passage 30 (light blue bars) and 50 (green bars) in WT controls and SAA cell lines. One-way ANOVA with Tukey's multiple comparison test was used for statistical comparison between fibroblasts and iPSC passage 30 and passage 50; d Analysis of telomerase activity in iPSC passage 50 (green bars) and iPSC-based hematopoietic progenitors (HPC) differentiated from iPSC passage 50 (light grey bars) in WT and SAA cell lines. Multiple $t$-test using Holm-Sidak method was used for statistical comparison between PSC passage 50 and HPC. a-d data is presented as mean of at least 3 independent experiments \pm S.E.M. Data for all control cell lines is averaged in one group (WT) 
which were significantly shorter than parent fibroblasts. The control iPSC lines did not show a significant telomere shortening during the 12 day differentiation time course to hematopoietic lineages; however all SAA-iPSC lines displayed a significant telomere attrition during the differentiation process, resulting in iPSC-derivedhematopoietic progenitors with significantly shorter telomeres than undifferentiated iPSC (Fig. 4b),corroborating data obtained with AA patient specific peripheral blood and bone marrow nucleated cells ${ }^{26,28-30}$.

We assessed telomerase activity in iPSC and iPSCderived-hematopoietic progenitors by telomere repeat amplification analysis. Telomerase activity was significantly increased in iPSC when compared to parental fibroblasts indicating the expected up-regulation of telomerase activity during reprogramming of both control and SAA fibroblasts as reported from other studies ${ }^{31}$ (Fig. 4c). Likewise, analysis of telomerase activity in iPSCderived-hematopoietic progenitors revealed no significant differences in telomerase activity compared to undifferentiated iPSC (Fig. 4d). Together, these data indicate impaired telomere elongation during the reprogramming of SAA fibroblasts, independent of telomerase activity measured by in vitro assays.

\section{Reduced proliferation capacity of SAA-iPSC-derived hematopoietic progenitors}

Progressive telomere shortening leads eventually to cell cycle arrest or cell death ${ }^{32,33}$. In view of this as well as the reduced capacity of SAA-iPSC to give rise to erythroid and myeloid cells, we investigated the proliferative capacity and apoptosis of the SAA iPSC-derivedhematopoietic progenitors. We synchronized the iPSCderived-hematopoietic progenitors in G1/S phase by treatment with ribonucleotide reductase inhibitor hydroxyurea (HU) for $24 \mathrm{~h}$. This was followed by culture in HUfree media and pulsing with 5-bromo-2-deoxyuridine (BrdU) for $1 \mathrm{~h}$ (Fig. 5a). Flow cytometric analysis of DNA content and incorporation of BrdU in control-iPSCderived-hematopoietic progenitors indicated a high percentage of cells arrested in G1/S phase at 1 and $3 \mathrm{~h}$ postrelease from $\mathrm{HU}$ due to depletion of deoxyribonucleotide pools (Supplementary Fig. 5a). These arrested cells reentered the cell cycle, progressed through S-phase and showed a similar cell cycle profile to that of untreated cells at $24 \mathrm{~h}$ post treatment (Supplementary Fig. 5a, b), indicating that iPSC-derived-hematopoietic progenitors require at least $24 \mathrm{~h}$ to restore a normal cell-cycle profile. We analyzed the proliferation rate of the synchronized control and SAA-iPSC-derived-hematopoietic progenitors (marked by CD43 expression) by comparing the percentage of BrdU-incorporating cells (S-phase) at $24 \mathrm{~h}$ post-release from $\mathrm{HU}$. Interestingly, SAA-iPSC-derivedhematopoietic progenitors showed a significant reduction in the number of BrdU-incorporating cells (proliferative cells) compared with control counterparts, indicating a reduced proliferative capacity (Fig. 5b). To investigate whether apoptosis was increased in SAA-iPSC-derivedhematopoietic progenitors, we measured the presence of cleaved Poly (ADP-ribose) polymerase-1 (PARP) by flow cytometric analysis. Cleaved-PARP analysis of early apoptotic cells revealed a significant increase in the apoptosis of SAA-iPSC-derived-hematopoietic progenitors derived from one of the patients (SAA2) when compared to control cells (Supplementary Fig. 5c). However, no significant differences in apoptosis were observed between patients and controls after HU treatment (Supplementary Fig. 5d).

Induction of replicative stress by depletion of deoxyribonucleotide pools with long exposure to hydroxyurea leads to stalled replication forks and accumulation of DNA damage ${ }^{34}$. To address whether reduced hematopoietic potential of SAA-iPSC-derived-hematopoietic progenitors might be attributed to an impaired ability to repair DNA damage associated with replicative stress, we analyzed the percentage of DNA damage induced by $\mathrm{HU}$ treatment by flow cytometric analysis in proliferating and non-proliferating cells (Fig. 5c-f). To assess the reversibility of the DNA damage induced by HU exposure, we measured the formation of the phosphorylated histone variant $\mathrm{H} 2 \mathrm{AX}(\gamma \mathrm{H} 2 \mathrm{AX})$ at $1,3,8$, and $24 \mathrm{~h}$ after $\mathrm{HU}$ release in control-iPSC-derived-hematopoietic progenitors. This analysis indicated accumulation of $\gamma \mathrm{H} 2 \mathrm{AX}+$ in $\mathrm{BrdU}+$ cells at 1 and $3 \mathrm{~h}$ after $\mathrm{HU}$ release indicating accumulation of DNA damage in replication forks after HU replication block (Supplementary Fig. 5e). The $\gamma \mathrm{H} 2 \mathrm{AX}$ foci progressively disappeared showing similar levels of $\gamma \mathrm{H} 2 \mathrm{AX}$ levels to those of untreated cells at $24 \mathrm{~h}$ (Supplementary Fig. 5e). Thus, analysis of accumulation of DNA damage in proliferating $(\gamma \mathrm{H} 2 \mathrm{AX}+\mathrm{BrdU}+)$ and non-proliferating $(\gamma \mathrm{H} 2 \mathrm{AX}+\mathrm{BrdU}-) \quad$ iPSC-derivedhematopoietic progenitors showed that control cell lines were able to repair the induced DNA damage after $24 \mathrm{~h}$ release from HU (Supplementary Fig. 5f, g).

To investigate the ability of SAA cell lines to repair DNA damage associated to replicative stress, we then analyzed the accumulation of $\gamma \mathrm{H} 2 \mathrm{AX}$ in proliferating $(\mathrm{BrdU}+)$ and non-proliferating (BrdU-) in SAA-iPSCderived-hematopoietic progenitors at 1 and $24 \mathrm{~h}$ after $\mathrm{HU}$ release (Fig. $5 \mathrm{c}-\mathrm{f}$ ). No significant increase in the accumulation of $\gamma \mathrm{H} 2 \mathrm{AX}+\mathrm{BrdU}+$ was observed at $1 \mathrm{~h}$ post $\mathrm{HU}$ release in SAA-iPSC-derived hematopoietic progenitors (Fig. 5c), unlike the control counterparts which showed a significant accumulation of DNA damage at $1 \mathrm{~h}$ post $\mathrm{HU}$ release. Low levels of DNA damage observed in BrdU+ after replicative stress points to a reduced proliferation capacity likely due to a reduced formation of replication forks, in agreement with our results showing significantly 
a

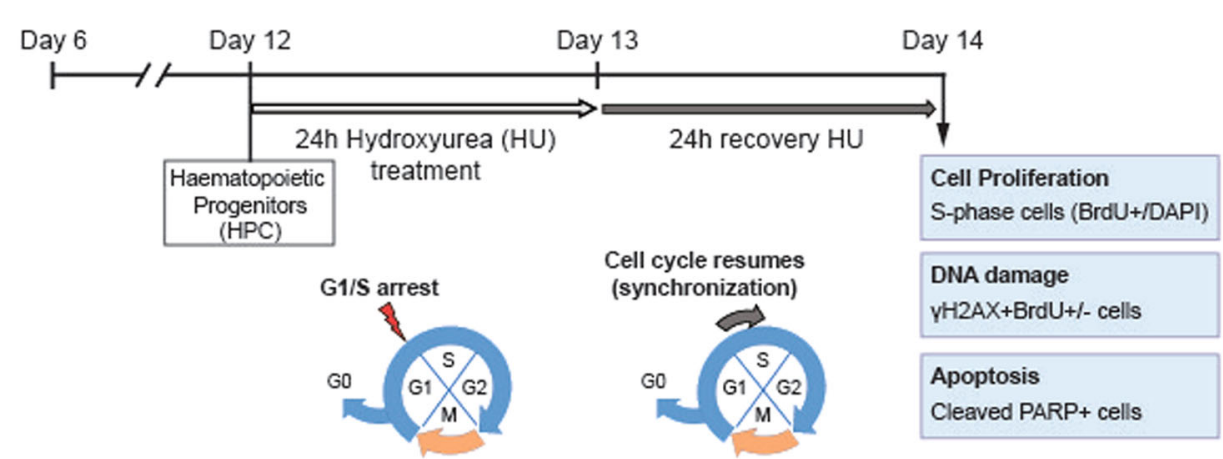

b

BrdU+ 24h HU recovery HPC

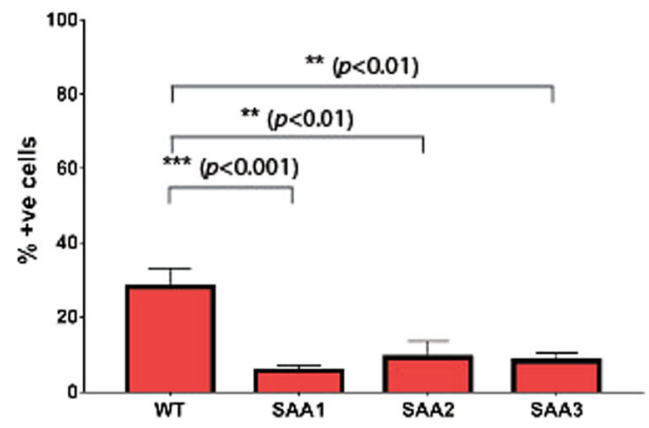

C

$\gamma \mathrm{H} 2 \mathrm{AX}+\mathrm{BrdU}+$

d

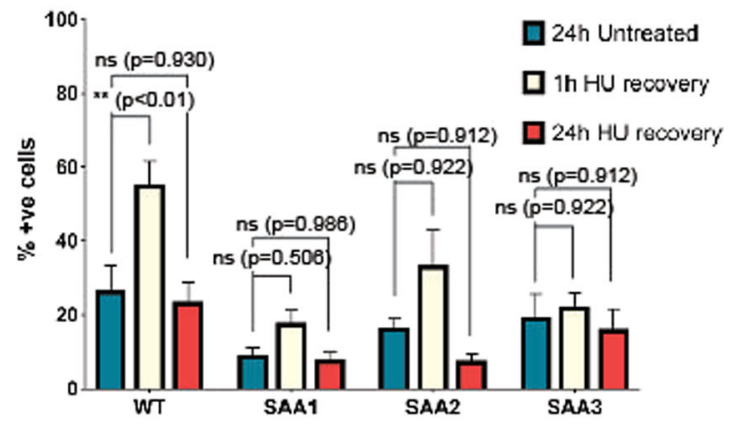

$\mathrm{YH} 2 \mathrm{AX}+\mathrm{BrdU}+$

e

$y H 2 A X+B r d U-$
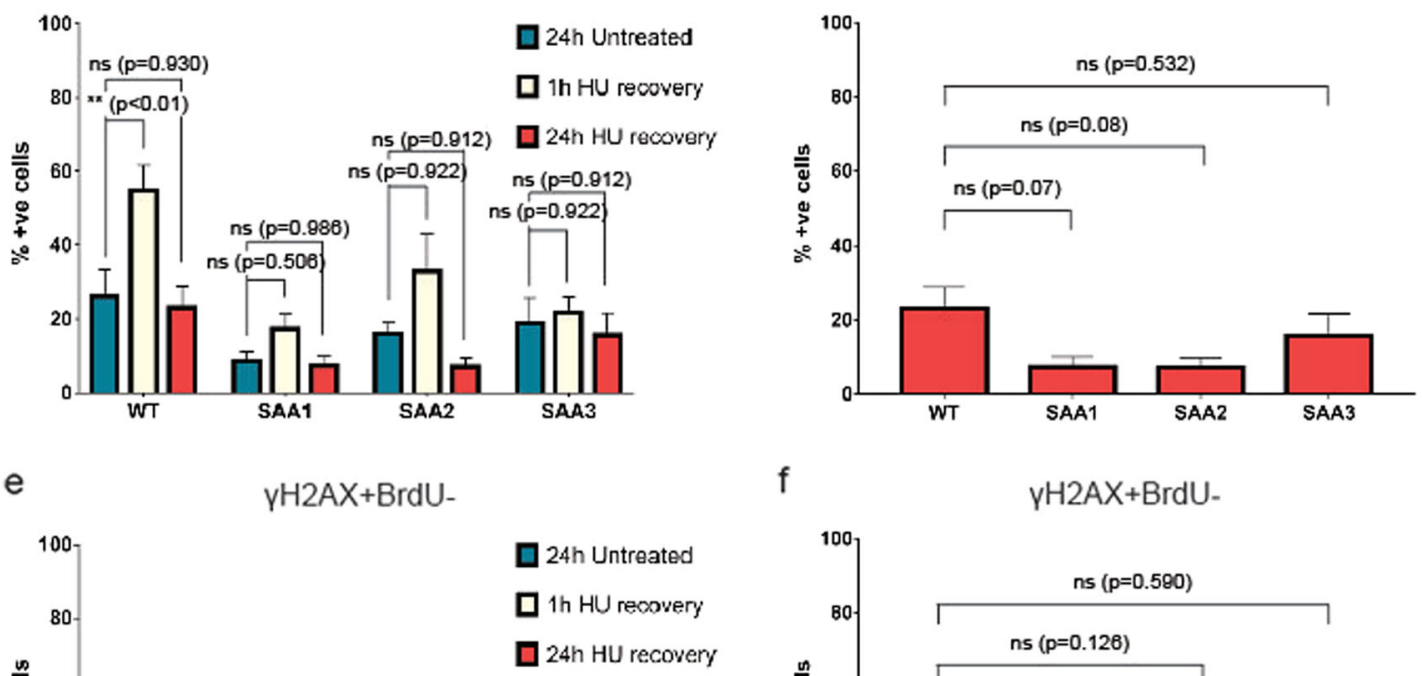

f

$\gamma \mathrm{H} 2 \mathrm{AX}+\mathrm{BrdU}-$

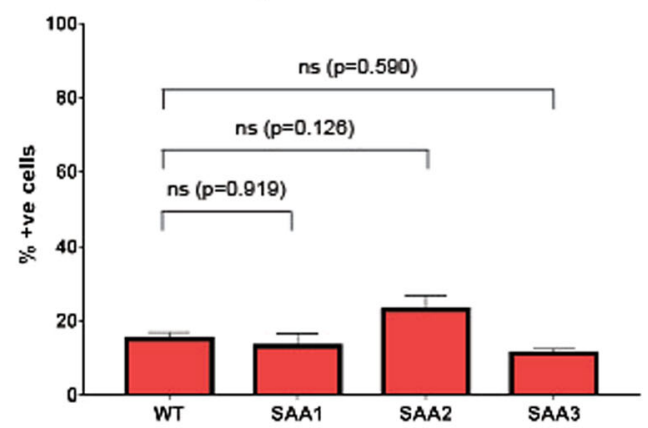

Fig. 5 (See legend on next page.) 
(see figure on previous page)

Fig. 5 SAA-iPSC-derived-hematopoietic progenitors show a reduced proliferation capacity. a Schematic of the experimental design used to analyze the proliferation, DNA repair capacity and apoptosis in SAA-iPSC-derived-hematopoietic progenitors; $\mathbf{b}$ Analysis of BrdU-incorporating cells in WT and SAA iPSC-derived-hematopoietic progenitors. One-way ANOVA with Dunnett's multiple comparison test was used for statistical comparison between WT and SAA cell lines; c Analysis of $\mathrm{YH} 2 \mathrm{AX}$ in BrdU+ cells in untreated (dark blue bars), $1 \mathrm{~h}$ after HU recovery (beige bars) and $24 \mathrm{~h}$ after HU recovery (red bars) iPSC-derived-hematopoietic progenitors. One-way ANOVA with Tukey's multiple comparison test was used for statistical comparison between untreated cells and $1 \mathrm{~h}$ after $\mathrm{HU}$ recovery and $24 \mathrm{~h}$ after recovery; d Analysis of YH2AX in BrdU+ cells in WT and SAA iPSCderived-hematopoietic progenitors. One-way ANOVA with Dunnett's multiple comparison test was used for statistical comparison between WT and SAA cell lines; e Analysis of YH2AX in BrdU- cells in untreated (dark blue bars), $1 \mathrm{~h}$ after $\mathrm{HU}$ recovery (beige bars) and $24 \mathrm{~h}$ after HU recovery (red bars) iPSC-derived-hematopoietic progenitors. One-way ANOVA with Tukey's multiple comparison test was used for statistical comparison between untreated cells and $1 \mathrm{~h}$ after $\mathrm{HU}$ recovery and $24 \mathrm{~h}$ after recovery, $\left({ }^{*} p<0.05\right)$; $\mathbf{f}$ Analysis of $\gamma \mathrm{H} 2 \mathrm{AX}$ in BrdU- cells in WT and SAA iPSC-derivedhematopoietic progenitors. One-way ANOVA with Dunnett's multiple comparison test was used for statistical comparison between WT and SAA cell lines. b-f data is presented as mean of at least 3 independent experiments \pm S.E.M. Data for all control cell lines is averaged in one group (WT)

reduced number of BrdU-incorporating cells (Fig. 5b). Thus, higher levels of $\gamma \mathrm{H} 2 \mathrm{AX}+\mathrm{BrdU}+$ cells were observed in control-iPSC-derived-hematopoietic progenitors compared to those in SAA cell lines, likely due to a higher number of replication forks, although the differences were not statistically significant (Fig. 5d). Interestingly, analysis of DNA damage in in non-proliferating (BrdU-) progenitors revealed a significant increase in the level of $\gamma \mathrm{H} 2 \mathrm{AX}$ at $24 \mathrm{~h}$ post $\mathrm{HU}$ release in one of patientderived-hematopoietic progenitors (SAA2) compared to equivalent cells generated from the controls (Fig. 5e), suggesting an individual patient specific impaired ability to restore normal levels of DNA damage after HU treatment in non-proliferating iPSC-derived-hematopoietic progenitors. Notwithstanding this, the percentage of $\gamma \mathrm{H} 2 \mathrm{AX}+\mathrm{BrdU}-\mathrm{cells}$ in the hematopoietic progenitors derived from this patient iPSC line was not significantly higher when compared to equivalent cells generated from the unaffected controls (Fig. 5f). Together these data indicate that proliferating SAA-iPSC-derivedhematopoietic progenitors tend to accumulate less DNA damage soon after HU treatment most likely as result of their reduced proliferation. Furthermore, a subset of SAA-iPSC-derived-hematopoietic progenitors may be slower or have an impaired ability to restore DNA damage in the non-proliferative compartment. However, the overall level of DNA damage induced in response to replicative stress is not significantly different compared to control-derived-hematopoietic progenitors, excluding DNA damage accumulation as a key factor underlying the impaired hematopoietic differentiation of SAA-iPSC lines.

\section{Eltrombopag does not improve hematopoietic differentiation potential or enhance proliferation of SAA-iPSC-derived-hematopoietic progenitors}

Eltrombopag (EP) is a thrombopoietin (TPO) receptor agonist that promotes megakaryocyte maturation and platelet production without competing with endogenous $\mathrm{TPO}^{35}$. Beyond its role in megakaryopoiesis and platelet generation, TPO signaling has been also shown to be critical for HSC homeostasis and expansion in animal models and refractory SAA patients ${ }^{36-38}$. In view of these findings, we investigated whether the observed reduced colony-forming potential and proliferation of SAA-iPSCderived-hematopoietic progenitors could be rescued by the addition of EP $(10 \mu \mathrm{M})$ to the differentiation media (Fig. 6a). Addition of EP to TPO containing differentiation media induced a significant increase in the percentage of erythroid progenitors $(\mathrm{CD} 43+\mathrm{CD} 235 \mathrm{a}+\mathrm{CD} 41 \mathrm{a}-)$ at the expense of megakaryocytic progenitors $(\mathrm{CD} 43+\mathrm{CD} 235 \mathrm{a}-$ $\mathrm{CD} 41+$ ) compared to the control group containing only TPO in differentiation media (data not shown). These results are very similar to what has been reported in CD34 + bone marrow cells ${ }^{39}$ and indicate that EP is biologically active in our iPSC differentiation system.

To assess EP's impact on SAA-iPSC differentiation, we added EP from day 6 of differentiation (Fig. 6a) in the presence and absence of $\mathrm{HU}$ and performed CFU assays at day 14 of differentiation. CFU assay results did not show significant differences between DMSO and EP-treated groups in the total number of colonies generated from SAA-iPSC-derived-hematopoietic progenitors cultured in absence of HU (Fig. 6b). Similarly, no differences were observed when EP was added in parallel to replicative stress inducing agent, HU (Fig. 6c).

EP favors the proliferation and DNA double-strand break repair after $\gamma$-irradiation in human hematopoietic stem and progenitor cells ${ }^{40,41}$. To investigate whether EP is affecting the proliferative and DNA damage repair capacity of the SAA-iPSC-derived-hematopoietic progenitors under conditions of replicative stress induced by $\mathrm{HU}$, flow cytometric analysis for BrdU incorporation and accumulation of $\gamma \mathrm{H} 2 \mathrm{AX}$ was carried out after EP treatment. No significant differences were observed in the percentage of BrdU+ cells, indicating that EP does not affect the proliferation of control or SAA-iPSC-derived 


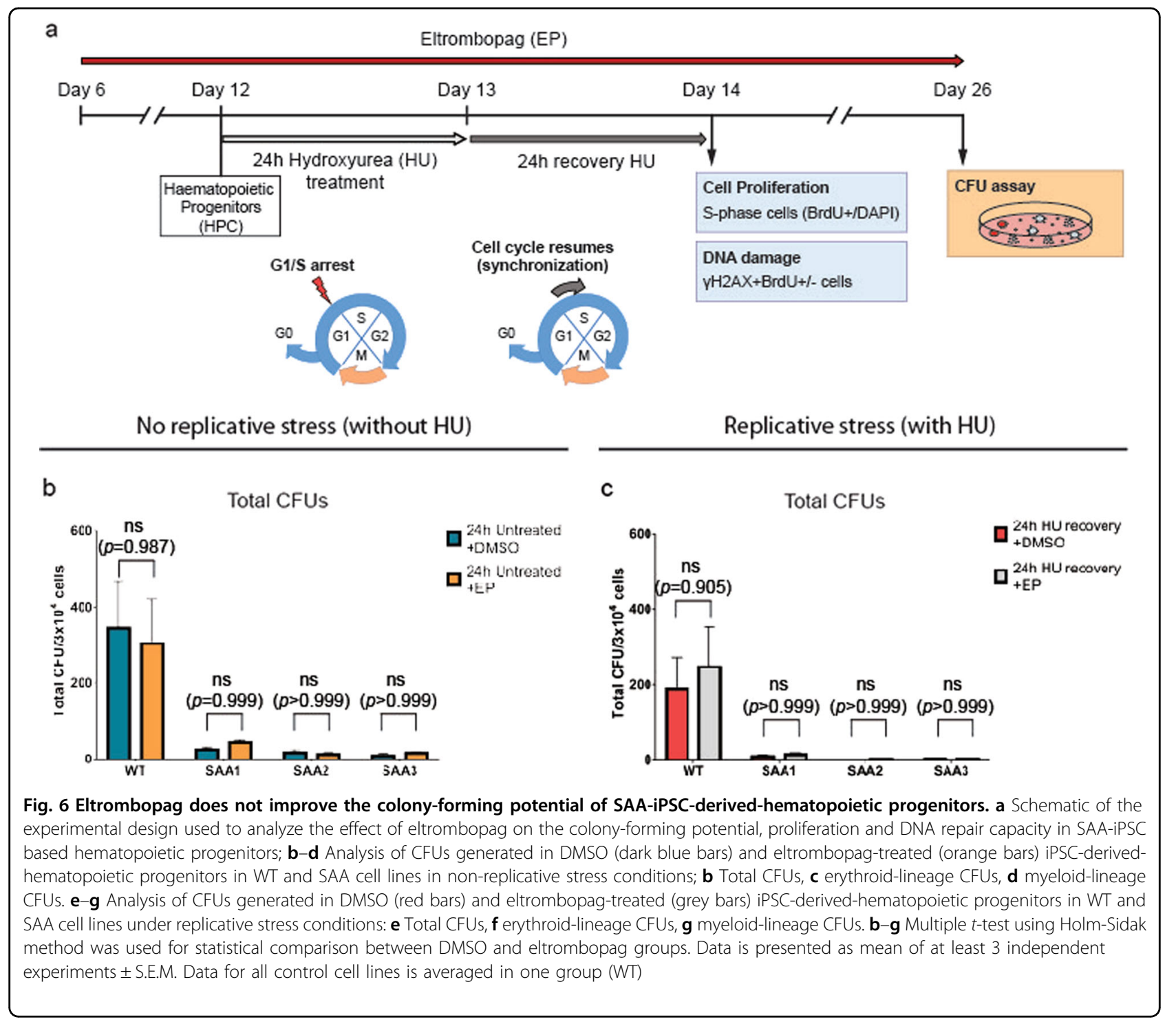

hematopoietic progenitors (Fig. 7a). Similarly, no significant changes were observed in the percentage of proliferating and non-proliferating control-and SAAiPSC-derived-hematopoietic progenitors with $\gamma \mathrm{H} 2 \mathrm{AX}$ foci (Fig. 7b, c). Together these data indicate that EP does not affect the proliferative capacity, DNA repair ability and colony forming potential of SAA-iPSC-derivedhematopoietic progenitor cells.

\section{Discussion}

Studies of hematopoietic progenitor cell dysfunction in SAA have been difficult due to paucity of these cells in patient's bone marrow samples. We have overcome this difficulty by using an iPSC disease model of three patients with SAA which has enabled us to study the phenotype of hematopoietic progenitors independent of the influence of the immune system.
SAA-iPSC lines were largely not impaired in their ability to generate hematopoietic progenitors; however the latter exhibited a much reduced erythroid and myeloid CFU ability accurately recapitulating the phenotype that defines SAA. The presence of reduced number and clonogenic capacity of bone marrow hematopoietic progenitors in AA patients has been reported by different studies $^{4,42,43}$ and traditionally considered secondary to immune-mediated stem cell destruction ${ }^{24}$. Using our in vitro SAA iPSC model, we provide evidence for an impaired differentiation capacity in SAA hematopoietic progenitors in the absence of an immune system, which may be suggestive of a constitutional progenitor cell dysfunction. Genetic background has been reported as one of the major drivers of phenotypic variability in iPSC modeling ${ }^{44-48}$. We minimized this effect in the SAA-iPSC model by including multiple control cell lines in order to 


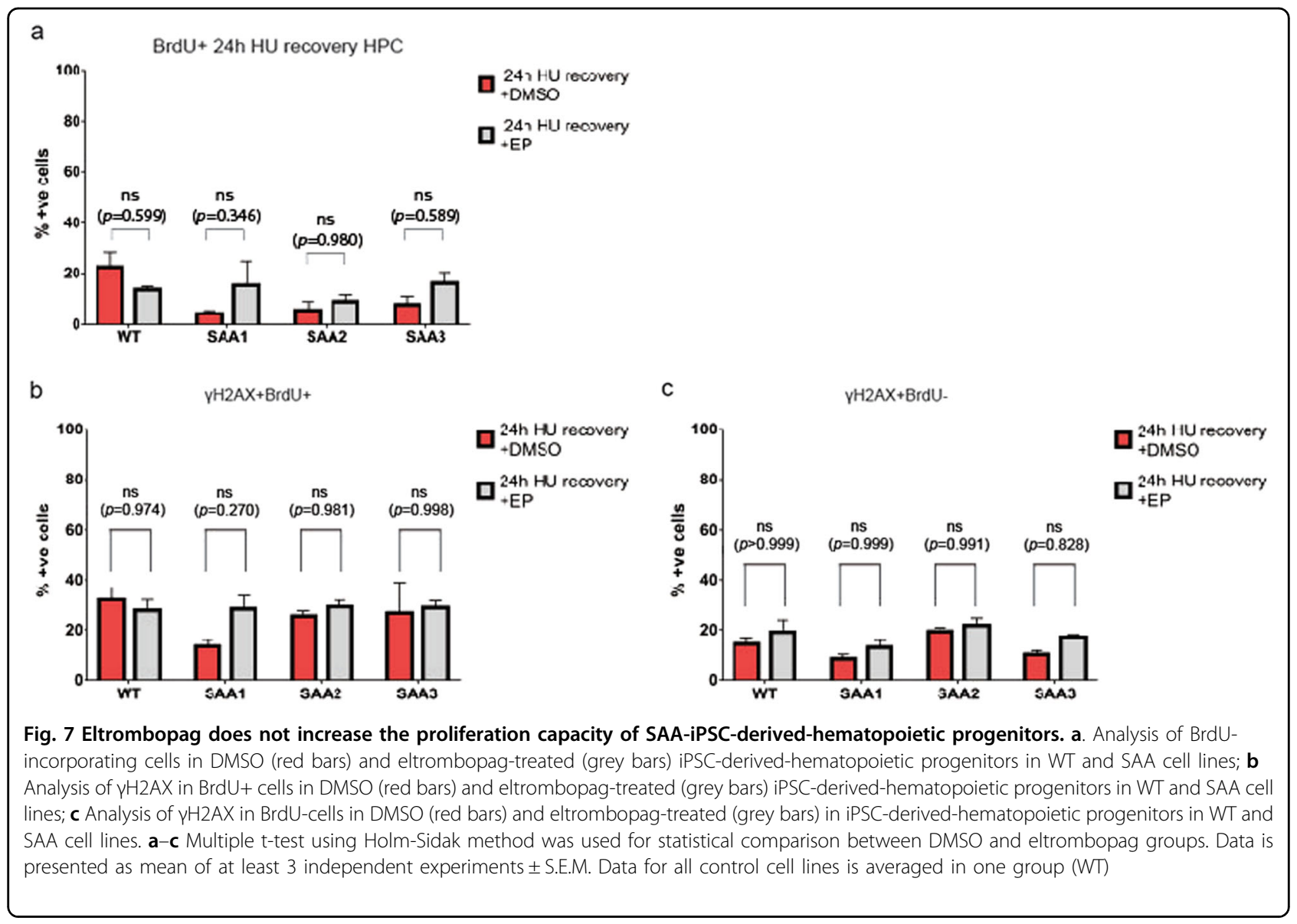

distinguish between phenotypic effects caused by diseasecausing alterations and genetic background. Further experiments using a larger number of iPSC lines from relatives sharing similar genetic background are needed to fully understand the disease pathogenesis.

Functional reconstruction of telomeres and upregulation of telomerase activity during reprogramming represent a hallmark of induced pluripotency ${ }^{27,31,49}$. iPSCderived modeling of telomeropathies associated with BMFs caused by mutations in telomerase-associated genes such as TERC, TERT, and DKC have shown defective telomere elongation in iPSC due to reduced telomerase function which impacts on the maintenance of pluripotent phenotype and hematopoietic differentiation capacity $^{50-52}$. In SAA, only $10 \%$ of patients with short telomeres display known mutations in telomere pathway components suggesting that mutations in uncharacterized genes might have a role in the disease phenotype observed in these patients ${ }^{24}$. Our results revealed that SAA fibroblasts failed to elongate telomeres during the reprogramming process despite up-regulation of telomerase activity, pointing to an impaired telomere elongation during the reprogramming process that is not attributable to a defective telomerase activity. Similarly, iPSC-based hematopoietic progenitors in SAA cell lines showed excessive telomere shortening during hematopoietic differentiation. It is possible that the presence of short telomeres may be responsible for the impaired hematopoietic differentiation capacity we observed in our iPSC derived modeling of SAA ${ }^{51}$. Alternatively, the short telomeres can be a consequence of reiterative rounds of divisions carried out by a smaller number of proliferating hematopoietic progenitor cells in SAA patients as a compensatory mechanism to maintain homeostasis ${ }^{15}$. To be able to distinguish between these possibilities and investigate whether genetic predispositions play a role, whole genome analysis of a large number of SAA patients and their families need to be carried out. Our study included three patients only which precludes such large scale analysis, however exome sequencing analysis of patient's dermal fibroblasts revealed the presence of deleterious mutations in telomere-associated genes such as RPA2, NCL, POLD3, TEP1, YLMP1, PIF1, and ERCC4 (Supplementary Table 3). Interestingly, replication Protein A (RPA) has been reported to be necessary for telomere maintenance due to its role in unfolding of 
telomeric G-quadruplexes and preventing replication-fork stalling $^{53}$ whereas $N C L$ codifies for the RNA chaperone nucleolin that regulates the nuclear localization of telomerase $^{54}$. Likewise, overexpression of Ylpm1 in mouse embryonic stem cells leads to down-regulation of telomerase activity and telomere shortening resulting in reduced proliferation and hematopoietic differentiation ability $^{55}$. Whilst these data point to existence of potentially deleterious mutations in telomere-associated genes in SAA patients, access to DNA samples from their related family members and larger cohorts of SAA patients is needed to confirm their involvement in SAA etiology.

EP is a non-peptide molecule mimetic to TPO that stimulates tri-lineage hematopoiesis in $40 \%$ of the SAA patients at $3-4$ months ${ }^{38,56}$. However, the mechanism by which EP is promoting the generation of blood cells and impacting hematopoietic stem and progenitors in AA patients is not fully understood. We studied the ability of EP to rescue the impaired hematopoietic differentiation capacity in our SAA-iPSC model. SAA-iPSC-derived hematopoietic progenitors did not show a significant increase in the number of erythroid or myeloid-lineage CFUs, proliferative capacity or DNA damage repair capacity under conditions of replicative stress, upon adding EP during the differentiation process. Interestingly, it has been reported that EP failed to improve severe thrombocytopenia in patients with constitutional bone marrow failures syndromes including DC and Diamond-Blackfan-anemia ${ }^{57}$. Thus, this lack of response to EP by DC patients supports our hypothesis that constitutional defects in the telomereassociated genes may be at the root of the impaired hematopoietic differentiation observed in the SAA-iPSCderived hematopoietic progenitors. As such it would be of interest to investigate if this group of patients will respond to other available therapies, for example danazol, although to date is unclear whether danazol related improvements are due to upregulation of telomerase activity through an increase in TERT expression or to elongation of telomeres $^{58,59}$. Likewise, different authors have hypothesized that EP might be involved in immune cell function by modulating regulatory $\mathrm{T}$ cell function in SAA patients $\mathrm{s}^{38,60}$ as observed in chronic idiopathic thrombocytopenic purpura patients treated with $\mathrm{EP}^{61}$. The lack of response to $\mathrm{EP}$ observed in our SAA-iPSC model suggests that EP may indeed have an underlying immune-regulatory function, which is separate to the impaired differentiation and telomere maintenance defect reported herein. In summary, our data provide strong evidence for usefulness of iPSC based disease modeling to replicate key phenotypes associated with SAA, facilitating the diagnosis of previously unidentified cases of constitutional SAA, and predicting patient specific response to various treatment modalities.

\section{Material and methods}

\section{Generation of iPSC lines from SAA patients and healthy volunteers}

Human fibroblasts from three healthy volunteers, one neonatal (Lonza, CC-2509) and two adult (Lonza, CC2511), and three patients with pediatric SAA were cultured with Advanced Dulbecco's Modified Eagle Medium (Thermo-Fisher, Waltham, MA, USA) containing 10\% FBS (Thermo Fisher Scientific), 1\% Glutamax (Thermo Fisher Scientific) and $1 \%$ penicillin/streptomycin (Thermo Fisher Scientific) at $37^{\circ} \mathrm{C}$ and $5 \% \mathrm{CO}_{2}$ in a humidified incubator. Fibroblasts were transduced using the Cytotune ${ }^{\mathrm{TM}}$-iPS Reprogramming Kit (Thermo Fisher Scientific, A1378001) according to manufacturer's instructions. iPSC colonies were established on inactivated primary mouse embryonic fibroblasts feeder layer and then adapted to a feeder-free system, cultured on recombinant Vitronectin (Thermo Fisher Scientific) and in StemPro hESC SFM ${ }^{\odot}$ media (Thermo Fisher Scientific) supplemented with 8ng/ ml basic Fibroblast Growth Factor (Thermo Fisher Scientific), $1 \%$ penicillin/streptomycin and $0.1 \mathrm{mM} 2$ mercaptoethanol (Thermo Fisher Scientific).

\section{In vitro test of pluripotency}

For immunocytochemistry analysis, iPSC colonies were fixed in $4 \%$ Formaldehyde (Sigma-Aldrich) and permeabilised with $0.25 \%$ Triton-X-100 (Sigma-Aldrich). Following treatment, cells were stained with mouse antihuman SSEA4-Alexa Fluor 555 (Beckton Dickinson, BD; Franklin Lakes, NJ, USA, 560218) at 1:60 dilution, mouse anti-human TRA-1-60-FITC (Merck Millipore, Billerica, MA, USA, FCMAB115F) at 1:60 dilution, mouse antiNANOG-AF647 (Cell Signaling Technologies, Danvers, MA, USA, 5448) at 1:150 dilution and goat antihuman OCT4 primary antibody (R\&D, Minneapolis, MN, USA, AF1759) at 1:60 dilution. Secondary staining was performed using anti-goat IgG FITC (Sigma-Aldrich, F7367) at 1:200 dilution. Following treatment with the nuclear stain-DAPI (Partec), stained iPSC colonies were photographed using a Bioscience Axiovert microscope (Axiocam, CarlZeiss) in combination with the associated CarlZeiss software- AxioVision.

To assess the percentage of cells expressing the pluripotent markers TRA-1-60 and SSEA-4, flow cytometric analysis was performed, iPSC colonies were dissociated using TrypLE ${ }^{\text {TM }}$ Express (Thermo Fisher Scientific) for 5 min at $37^{\circ} \mathrm{C}$. Dissociated cells were stained with the following antibodies: anti-human TRA-1-60-FITC (Millipore, FCMAB115) at 1:60 dilution and mouse anti-human SSEA-4-PerCPCy ${ }^{\mathrm{TM}} 5.5$ (BD, 561565) at 1:20. Cell population was identified based on cell size and cell granularity. Single cells were discriminated using Forward Scatter area (FSC-A) and Forward Scatter Height (FSC-H) and live 
cells were gated from single cell population using DAPI nuclear staining (Partec). The cells were acquired using the BD LSRII flow cytometer (BD) and data analyzed using FlowJo software (Tree Star, Ashland, OR, USA). At least 10.000 events were collected for each analysis.

\section{In vivo test of pluripotency}

For in vivo analysis of pluripotency via teratoma formation, $0.5 \times 10^{6}$ iPSCs were resuspended in $50 \%$ Matrigel $^{\mathrm{TM}}$ (BD, 356234) and injected subcutaneously into both flanks of adult SCID male mice. Two animals were injected in each group. Following a period of 10 weeks, the mice were euthanized and the teratomae were excised, processed and sectioned according to standard procedures and stained for Weigert's hematoxylin, Masson's trichrome and Mayer's hematoxylin and Eosin histological analysis. Sections $(5-8 \mu \mathrm{m})$ were examined using bright field microscopy and stained tissue photographed as appropriate.

\section{Karyotyping and fingerprinting analysis}

All cell lines were analyzed using Illumina CytoSNP analysis and the BlueFuse Multi 4.3 software (Illumina, San Diego, United States) according to standard protocols of the manufacturer.

\section{iPSC differentiation into hematopoietic progenitor cells}

iPSCs maintained on Vitronectin ${ }^{\mathrm{TM}}$ in StemPro ${ }^{\mathrm{TM}}$ media were cut in homogeneous pieces using a $\mathrm{STEMPRO}^{\circ}$ EZpassage $^{\mathrm{TM}}$ tool (Thermo Fisher Scientific). Aggregates were resuspended in Stemline ${ }^{\circ}$ II (Sigma-Aldrich) differentiation media supplemented with $1 \%$ penicillin/streptomycin and cultured in ultra-low attachment culture plates at $37{ }^{\circ} \mathrm{C}$ and $5 \% \mathrm{CO}_{2}$ in a humidified incubator for 3 days to allow the formation of embryoid bodies (EBs). On day 3, EBs were dissociated using TrypLE ${ }^{\mathrm{TM}}$ Express for $10 \mathrm{~min}$ at $37^{\circ} \mathrm{C}$ and transferred to tissue-culture treated wells to allow culture under monolayer conditions at $37^{\circ} \mathrm{C}$ and $5 \% \mathrm{CO}_{2}$ in a humidified incubator. Recombinant human BMP4 (day 0-2 $10 \mathrm{ng} / \mathrm{ml}$, day 2-16 $20 \mathrm{ng} / \mathrm{ml}$ ), VEGF (day 0-2 $10 \mathrm{ng} / \mathrm{ml}$, day

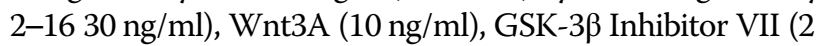
$\mu \mathrm{M})$, Activin A (5 ng/ml), FGF $\alpha(10 \mathrm{ng} / \mathrm{ml})$, SCF $(20 \mathrm{ng} / \mathrm{ml})$, IGF-2 $(10 \mathrm{ng} / \mathrm{ml})$, TPO $(10 \mathrm{ng} / \mathrm{ml}), \beta$-estradiol $(0.4 \mathrm{ng} / \mathrm{ml})$, Heparin $(5 \mu \mathrm{g} / \mathrm{ml})$ and 3-isobutyl-1-methylxanthine (IBMX) $(50 \mu \mathrm{M})$ were added to the differentiation media as previously described ${ }^{18}$. All cytokines and compounds were purchased from Peprotech except BMP4 and Wnt3A (R\&D), GSK-3 $\beta$ Inhibitor VII (Calbiochem) and $\beta$-estradiol, Heparin and IBMX (Sigma-Aldrich).

\section{Detection of mesodermal and hematopoietic markers by flow cytometry}

Differentiated cells were treated with $1 \mathrm{X}$ TrypLE ${ }^{\mathrm{TM}}$ Express for $5 \mathrm{~min}$ at $37^{\circ} \mathrm{C}$ to obtain a single a cell suspension. Cell pellets were resuspended in FACS buffer (DPBS with FBS 2\%) and cells were counted with a hemocytometer. A final amount of $1 \times 10^{5}$ cells resuspended in $100 \mu \mathrm{l}$ of FACS buffer with a dilution of 1:20 antibody was used for each analysis. The following cell surface antigens were analyzed for this study: KDR-PE (BD, 560494), CD34-APC (BD, 555824), CD43-FITC (Thermo Fisher Scientific, MHCD4301), CD41a-APCH7 (BD, 561422) and CD235a-BV421 (BD, 562938). Cells were washed using the BD FACS Lyse/Wash assistant (BD) and analyzed using BD LSRII flow cytometer (BD). Size and cell complexity were used to identify cell populations in a scatter graph representation. Single cells were discriminated using FSC-A and FSC-H and live cells were gated from single cell population using DAPI nuclear staining. Analysis of data was performed using FlowJo software (Tree Star Inc.). At least 10.000 events were collected for each analysis.

\section{Analysis of hematopoietic potential of hematopoietic progenitors by colony-forming unit assay}

iPSC-derived hematopoietic progenitor cells were treated with $1 \mathrm{X}$ TrypLE ${ }^{\mathrm{TM}}$ Express for $5-10 \mathrm{~min}$ at $37^{\circ} \mathrm{C}$ to obtain a single a cell suspension. TrypLE ${ }^{\mathrm{TM}}$ Express is diluted in PBS and cells were pelleted by centrifugation at $300 \mathrm{~g}$ for $3 \mathrm{~min}$. Cell pellets were resuspended in FACS buffer (DPBS with $2 \%$ FBS) and cells were counted with a hemocytometer. A final amount of $6 \times 10^{4}$ cells resuspended in $300 \mu \mathrm{l}$ of FACS buffer and mixed with $3 \mathrm{ml}$ of Methocult $^{\mathrm{TM}}$ methylcellulose media enriched with recombinant cytokines (Stem Cell Technologies, 04435) and $1.5 \mathrm{ml}$ were plated in duplicate in $35-\mathrm{mm}$ dishes. Colonies were scored after 14 days of culture using light microscope according to standard criteria and averaged between the duplicate dishes.

\section{RNA isolation and reverse transcription PCR (RT-PCR)}

RNA from iPSCs and fibroblasts at day-7 of $\mathrm{SeV}$ transduction used as positive Sendai control was extracted using the ReliaPrep ${ }^{\text {TM }}$ RNA Cell Miniprep System (Promega, Z6010) including DNase I treatment according to the manufacturer's instructions. cDNA was generated from $1 \mu \mathrm{g}$ of RNA using the GoScript ${ }^{\mathrm{TM}}$ Reverse Transcription System (Promega, A5000) according to the manufacturer's instructions.

For the PCR reaction mixture, $1 \mu \mathrm{l}$ of cDNA produced from $1 \mu \mathrm{g}$ of RNA was amplified using $10 \mu \mathrm{M}$ dNTP mix, 5X Green GoTaq ${ }^{\circ}$ Reaction Buffer and GoTaq ${ }^{\circ}$ DNA Polymerase $(5 \mathrm{u} / \mu \mathrm{l})$ (Promega, M3175) and the following primers $(10 \mu \mathrm{M}) \mathrm{SeV}$-OCT4 forward: 5'-CCCGAAAGAGAA AGCGAACCAG-3'; $\mathrm{SeV}$-OCT4 reverse: 5'-AATGTATCGA AGGTGCTCAA-3'; $\mathrm{SeV}$-SOX2 forward: 5'-ATGCACCGCTACGACGTGAGCGC-3'; SeV-SOX2 reverse: 5'-AATGTATCGAAGGTGCTCAA-3'; SeV-Klf4 forward: 5'-TTCCT 
GCATGCCAGAGGAGCCC-3'; SeV-Klf4 reverse: 5'-AATG TATCGAAGGTGCTCAA-3'; SeV-cMYC forward: $5^{\prime}$-TA ACTGACTAGCAGGCTTGTCG-3'; SeV-cMYC reverse: $5^{\prime}$ TCCACATACAGTCCTGGATGATGATG-3'; $\mathrm{SeV}$ forward: 5'-GGATCACTAGGTGATATCGAGC-3'; $\mathrm{SeV}$ reverse: 5'-ACCAGACAAGAGTTTAAGAGATATGTA TC-3'. Oligonucleotides for the housekeeping geneGAPDH were used as a positive control of the amplification reaction.

\section{DNA isolation and quantitative PCR for telomere length measurement}

Genomic DNA was isolated using QiAamp DNA Mini Kit (Qiagen) according to manufacturer's instructions. Telomere length was measured as abundance of telomeric template versus a single copy gene (36B4) as previously described $^{62}$ using the following primers: TelA (5'-CGG TTT GTT TGG GTT TGG GTT TGG GTT TGG GTT TGG GTT-3'); TelB (5'-GGC TTG CCT TAC CCT TAC CCT TAC CCT TAC CCT TAC CCT-3'); 36B4F (5'-CAG CAA GTG GGA AGG TGT AAT CC $\left.3^{\prime}\right)$ and 36B4R (5'CCC ATT CTA TCA TCA ACG GGT ACA A-3'). Three internal control DNA samples of known telomere length $(10.4,3.9$ and $2 \mathrm{~kb})$ were run within each plate to correct for plate-to-plate variation. Measurements were performed in triplicate. All PCRs were carried out on an Applied Biosystems 7900HT Fast Real Time PCR system with 384-well plate capacity. The intra-assay coefficient of variation was $2.7 \%$ while the inter-assay coefficient of variation was $5.1 \%$.

\section{Telomere repeat amplification analysis for telomerase activity detection}

Telomerase activity in iPSC and iPSC-derived hematopoietic progenitors was measured as previously described $^{63}$. Final amount of $100 \mathrm{ng}$ was used from protein lysate for each reaction. No protein/lysate samples were used as negative controls and 100, 10, 1, and $0.1 \mathrm{ng}$ of protein lysate from Hela cells as positive controls.

\section{Analysis of DNA damage, proliferation and apoptosis by flow cytometry}

Day 12 iPSC-derived hematopoietic progenitor cells were exposed to $2 \mathrm{M}$ Hydroxyurea for $24 \mathrm{~h}$ and collected at different time points $(0,1,3,8$, and $24 \mathrm{~h})$. DNA damage, proliferation and apoptosis induction after HU-treatment was analyzed using a flow cytometric kit according to manufacturer's instructions (BD). Briefly, at the specified time points after HU treatment, the cells were labeled with $50 \mu \mathrm{M}$ BrdU and stained later with antibody antihuman CD43-FITC. The labeled cells were then fixed, permeabilised and labeled with anti-human $\gamma \mathrm{H} 2 \mathrm{AX}$-Alexa Fluor ${ }^{\circ} 647$, anti-human BrdU- PerCPCy ${ }^{\mathrm{TM}} 5.5$ and antihuman Cleaved PARP (Asp214)-PE according to manufacturer's instructions. DNA content for cell cycle analysis was determined by DAPI staining provided by the kit. Size and cell complexity were used to identify cell populations in a scatter graph representation and single cells were discriminated using FSC area (FSC-A) and FSC height $(\mathrm{FSC}-\mathrm{H})$. The cells were acquired using the $\mathrm{BD}$ LSRII flow cytometer (BD) and data analyzed using FlowJo software. At least 10.000 events were collected for each analysis.

\section{Exome sequencing analysis}

DNA was extracted from 5 HLHS patient fibroblasts using QIAamp DNA Micro kit (Qiagen, Germantown, $\mathrm{MD}, 56304)$. Exome sequencing was performed by BGI. Exome capture was performed using Agilent SureSelect Human All Exon kit (V4). Libraries were constructed following the Illumina Paired-End Sequencing Library Preparation Protocol version 1.0.1 and then sequenced on the Illumina GAIIx platform with version 4 chemistry and version 4 flowcells. The sequencing reads were analyzed using the following workflow to identify variants in patient. The quality of sequencing reads was firstly checked with FastQC (Version 0.11.2) ${ }^{64}$ Low quality bases $(\mathrm{Q}<20)$ on $3^{\prime}$ ends of reads were trimmed off using seqtk $(\text { Version 1.0 })^{65}$. Duplicated reads were then removed with FastUniq (Version 1.1) ${ }^{66}$ before mapping to the human reference genome GRCh37 with BWA (Version 0.7.6.a) ${ }^{67}$. The alignments were refined with tools of the GATK suite (Version 3.2) ${ }^{68}$. Variants were called according to GATK Best Practice recommendations ${ }^{69,70}$, including recalibration. Freebayes (Version 1.0.1) ${ }^{71}$ was also used to call variants from the same set of samples. The variants called by Freebayes with total coverage $\geq 5$, minor allele coverage $\geq 5$ and variants call quality $\geq 20$ were added to those identified by GATK. Non-synonymous exonic variants were subsequently filtered by quality and minor-allele frequency (MAF) reported in the 1000 Genomes project $(2012 \mathrm{Feb} \text { release })^{72}$ and ESP6500 ${ }^{73}$. Variants with MAF $>0.05$ in either of the databases were excluded. ANNOVAR (Version 2014-07-22) ${ }^{74}$ was used for annotations and prediction of functional consequences. Deleterious in our study was defined by at least one of the predictors [SIFT or PolyPHEN2] and those that had alternative allele frequency less than 0.01 in both 1000 Genomes project and ESP6500 (Supplementary Table 2).

\section{Statistical analysis}

Data are shown as mean \pm S.E.M. from at least three independent experiments. The significance between means was determined with Multiple $t$-test using HolmSidak method and One-way ANOVA when Gaussian distribution was assumed and with Kruskal-Wallis test when Gaussian distribution was not assumed. Multiple comparisons test for comparison between particular pairs 
of control and patient groups. Statistically significant values were judged as follows: ${ }^{*} P \leq 0.05,{ }^{* * *} P \leq 0.01$, ${ }^{* * * *} P \leq$ 0.001 , $\cdots * \cdots P 0.0001$. Statistical analysis was performed using GraphPad Prism version 7.0 software and Minitab 17 statistical software.

\section{Acknowledgements}

The authors are grateful to ERC (\#614620), Great Ormond Street Hospital Charity (\#SL01A10), BBSRC (BB/I020209/1), Anthony Booth Trust and Deanship of Scientific Research (DSR) King Abdul Aziz University (grant number 1343287-1-HiCi) for their financial support. The authors would like to thank Dr Joanne Mountford for helping with hematopoietic differentiation protocol, Dr. Carmen Martin-Ruiz for performing the telomere length analysis and the flow cytometry facility for technical support.

\section{Author details}

'Institute of Genetic Medicine, Newcastle University, Newcastle, UK. ${ }^{2}$ The Ageing Biology Centre. Institute for Cell and Molecular Biosciences, Newcastle University, Newcastle, UK. ${ }^{3}$ Princess Al Jawhara Al-Brahim Center of Excellence in Research of Hereditary Disorders, King Abdulaziz University, Jeddah, Saudi Arabia. ${ }^{4}$ Department of Bioscience, Durham University, Durham, UK. ${ }^{5}$ Hematology Department, King Abdulaziz University, Jeddah, Saudi Arabia. ${ }^{6}$ Department of Medicine, Duke University Medical Center, Durham, USA. ${ }^{7}$ Department of Hematology, Great Ormond Street Hospital for Children NHS Foundation Trust, London, UK

\section{Conflict of interest}

The authors declare that they have no conflict of interest.

\section{Publisher's note}

Springer Nature remains neutral with regard to jurisdictional claims in published maps and institutional affiliations.

\section{Supplementary information}

The online version of this article https://doi.org/10.1038/s41419-017-0141-1 contains supplementary material.

Received: 28 July 2017 Revised: 22 October 2017 Accepted: 10 November 2017

Published online: 26 January 2018

\section{References}

1. Biswajit, $H$. et al. Aplastic anemia: a common hematological abnormality among peripheral pancytopenia. North Am. J. Med. Sci. 4, 384-388 (2012).

2. Zeng, Y. \& Katsanis, E. The complex pathophysiology of acquired aplastic anaemia. Clin. Exp. Immunol. 180, 361-370 (2015).

3. Schrezenmeier, H., Jenal, M., Herrmann, F., Heimpel, H. \& Raghavachar, A. Quantitative analysis of cobblestone area-forming cells in bone marrow of patients with aplastic anemia by limiting dilution assay. Blood $\mathbf{8 8}, 4474-4480$ (1996).

4. Maciejewski, J. P., Selleri, C., Sato, T., Anderson, S. \& Young, N. S. A severe and consistent deficit in marrow and circulating primitive hematopoietic cells (long-term culture-initiating cells) in acquired aplastic anemia. Blood $\mathbf{8 8}$, 1983-1991 (1996).

5. Philpott, N. J., Scopes, J., Marsh, J. C., Gordon-Smith, E. C. \& Gibson, F. M. Increased apoptosis in aplastic anemia bone marrow progenitor cells: possible pathophysiologic significance. Exp. Hematol. 23, 1642-1648 (1995).

6. Kakagianni, T. et al. A probable role for trail-induced apoptosis in the pathogenesis of marrow failure. Implications from an in vitro model and from marrow of aplastic anemia patients. Leuk. Res. 30, 713-721 (2006).

7. Zeng, W. et al. Gene expression profiling in CD34 cells to identify differences between aplastic anemia patients and healthy volunteers. Blood 103, 325-332 (2004).

8. Solomou, E. E. et al. Perforin gene mutations in patients with acquired aplastic anemia. Blood 109, 5234-5237 (2007)

9. Walne, A. J. et al. Exome sequencing identifies MPL as a causative gene in familial aplastic anemia. Haematologica 97, 524-528 (2012).
10. Shimada, $H$. et al. First case of aplastic anemia in a Japanese child with a homozygous missense mutation in the NBS1 gene (1171V) associated with genomic instability. Hum. Genet. 115, 372-376 (2004).

11. Calado, R. T. et al. Mutations in the SBDS gene in acquired aplastic anemia. Blood 110, 1141-1146 (2007).

12. Yamaguchi, $H$. et al. Mutations in TERT, the gene for telomerase reverse transcriptase, in aplastic anemia. N. Eng. J. Med. 352, 1413-1424 (2005).

13. Dufour, $C$. et al. Homozygosis for (12) CA repeats in the first intron of the human IFN-gamma gene is significantly associated with the risk of aplastic anaemia in Caucasian population. Br. J. Haematol. 126, 682-685 (2004).

14. Adam, S. et al. Concise review: getting to the core of inherited bone marrow failures. Stem Cell 35, 284-298 (2016).

15. Beier, F., Foronda, M., Martinez, P. \& Blasco, M. A. Conditional TRF1 knockout in the hematopoietic compartment leads to bone marrow failure and recapitulates clinical features of dyskeratosis congenita. Blood 120, 2990-3000 (2012).

16. Chen, J. Animal models for acquired bone marrow failure syndromes. Clin. Med. Res. 3, 102-108 (2005).

17. Fusaki, N., Ban, H., Nishiyama, A., Saeki, K. \& Hasegawa, M. Efficient induction of transgene-free human pluripotent stem cells using a vector based on Sendai virus, an RNA virus that does not integrate into the host genome. Proc. Jpn. Acad. Ser. B Phys. Biol. Sci. 85, 348-362 (2009).

18. Olivier, E. N. et al. High-efficiency serum-free feeder-free erythroid differentiation of human pluripotent stem cells using small molecules. Stem Cells Transl. Med. 5, 1394-1405 (2016).

19. Wang, Y. \& Nakayama, N. WNT and BMP signaling are both required for hematopoietic cell development from human ES cells. Stem Cell Res. 3, 113-125 (2009).

20. Vodyanik, M. A., Thomson, J. A. \& Slukvin, I. I. Leukosialin (CD43) defines hematopoietic progenitors in human embryonic stem cell differentiation cultures. Blood 108, 2095-2105 (2006).

21. Kennedy, M. et al. T lymphocyte potential marks the emergence of definitive hematopoietic progenitors in human pluripotent stem cell differentiation cultures. Cell Rep. 2, 1722-1735 (2012).

22. Kotini, A. G. et al. Functional analysis of a chromosomal deletion associated with myelodysplastic syndromes using isogenic human induced pluripotent stem cells. Nat. Biotechnol. 33, 646-655 (2015).

23. Calado, R. T. \& Young, N. S. Telomere maintenance and human bone marrow failure. Blood 111, 4446-4455 (2008).

24. Young, N. S., Calado, R. T. \& Scheinberg, P. Current concepts in the pathophysiology and treatment of aplastic anemia. Blood 108, 2509-2519 (2006).

25. Ball, S. E. et al. Progressive telomere shortening in aplastic anemia. Blood $\mathbf{9 1}$ 3582-3592 (1998).

26. Brummendorf, T. H., Maciejewski, J. P., Mak, J., Young, N. S. \& Lansdorp, P. M. Telomere length in leukocyte subpopulations of patients with aplastic anemia. Blood 97, 895-900 (2001).

27. Marion, R. M. et al. Telomeres acquire embryonic stem cell characteristics in induced pluripotent stem cells. Cell. Stem. Cell. 4, 141-154 (2009).

28. Sakaguchi, H. et al. Peripheral blood lymphocyte telomere length as a pre dictor of response to immunosuppressive therapy in childhood aplastic anemia. Haematologica 99, 1312-1316 (2014).

29. Lee, J. J. et al. Telomere length changes in patients with aplastic anaemia. Br. J. Haematol. 112, 1025-1030 (2001).

30. Park, H. S. et al. Telomere length and somatic mutation in aplastic anemiacorrelation with response to treatement. Blood 128, 1504 (2016).

31. Takahashi, K. et al. Induction of pluripotent stem cells from adult human fibroblasts by defined factors. Cell 131, 861-872 (2007).

32. Harley, C. B., Futcher, A. B. \& Greider, C. W. Telomeres shorten during ageing of human fibroblasts. Nature 345, 458-460 (1990).

33. Blasco, M. A. Telomeres and human disease: ageing, cancer and beyond. Nat Rev. Genet. 6, 611-622 (2005).

34. Petermann, E., Orta, M. L., Issaeva, N., Schultz, N. \& Helleday, T. Hydroxyureastalled replication forks become progressively inactivated and require two different RAD51-mediated pathways for restart and repair. Mol. Cell. 37, 492-502 (2010)

35. Desmond, R., Townsley, D. M., Dunbar, C. \& Young, N. S. Eltrombopag in aplastic anemia. Semin. Hematol. 52, 31-37 (2015).

36. Alexander, W. S., Roberts, A. W., Nicola, N. A., Li, R. \& Metcalf, D. Deficiencies in progenitor cells of multiple hematopoietic lineages and defective megakaryocytopoiesis in mice lacking the thrombopoietic receptor c-Mpl. Blood 87, 2162-2170 (1996). 
37. Qian, $H$. et al. Critical role of thrombopoietin in maintaining adult quiescent hematopoietic stem cells. Cell. Stem. Cell. 1, 671-684 (2007).

38. Desmond, R. et al. Eltrombopag restores trilineage hematopoiesis in refractory severe aplastic anemia that can be sustained on discontinuation of drug. Blood 123, 1818-1825 (2014).

39. Jeong, J. Y. et al. The non-peptide thrombopoietin receptor agonist eltrombopag stimulates megakaryopoiesis in bone marrow cells from patients with relapsed multiple myeloma. J. Hematol. Oncol. 8, 37 (2015).

40. Sun, H., Tsai, Y., Nowak, l., Liesveld, J. \& Chen, Y. Eltrombopag, a thrombopoietin receptor agonist, enhances human umbilical cord blood hematopoietic stem/primitive progenitor cell expansion and promotes multi-lineage hematopoiesis. Stem Cell Res. 9, 77-86 (2012).

41. Cheruku, P. S. C. A., Dunbar, C. E., Young, N. S. \& Larochelle, A. Thethrombopoietin receptor agonist eltrombopag has dna repair activity in human hematopoietic stem and progenitor cells. Blood 126, 2407 (2015).

42. Marsh, J. C., Chang, J., Testa, N. G., Hows, J. M. \& Dexter, T. M. The hematopoietic defect in aplastic anemia assessed by long-term marrow culture. Blood 76, 1748-1757 (1990)

43. Rizzo, S. et al. Stem cell defect in aplastic anemia: reduced long term cultureinitiating cells (LTC-IC) in CD34+ cells isolated from aplastic anemia patient bone marrow. Hematol. J. 3, 230-236 (2002).

44. Carcamo-Orive, I. et al. Analysis of transcriptional variability in a large human iPSC library reveals genetic and non-genetic determinants of heterogeneity. Cell. Stem. Cell. 20, 518-532 e9 (2017).

45. Choi, J. et al. A comparison of genetically matched cell lines reveals the equivalence of human iPSCs and ESCs. Nat. Biotechnol. 33, 1173-1181 (2015).

46. Feraud, O. et al. Donor dependent variations in hematopoietic differentiation among embryonic and induced pluripotent stem cell lines. PLOS. ONE. 11 e0149291 (2016)

47. Kyttala, A. et al. Genetic variability overrides the impact of parental cell type and determines ipsc differentiation potential. Stem Cell Rep. 6, 200-212 (2016).

48. Rouhani, F. et al. Genetic background drives transcriptional variation in human induced pluripotent stem cells. PLoS. Genet. 10, e1004432 (2014).

49. Takahashi, K. \& Yamanaka, S. Induction of pluripotent stem cells from mouse embryonic and adult fibroblast cultures by defined factors. Cell 126, 663-676 (2006).

50. Batista, L. F. et al. Telomere shortening and loss of self-renewal in dyskeratosis congenita induced pluripotent stem cells. Nature 474, 399-402 (2011).

51. Winkler, T. et al. Defective telomere elongation and hematopoiesis from telomerase-mutant aplastic anemia iPSCs. J. Clin. Invest. 123, 1952-1963 (2013).

52. Gu, B. W. et al. Impaired telomere maintenance and decreased canonical WNT signaling but normal ribosome biogenesis in induced pluripotent stem cells from X-linked dyskeratosis congenita patients. PLOS. ONE. 10, e0127414 (2015).

53. Kobayashi, Y. et al. Expression of mutant RPA in human cancer cells causes telomere shortening. Biosci. Biotechnol. Biochem. 74, 382-385 (2010).

54. Khurts, S. et al. Nucleolin interacts with telomerase. J. Biol. Chem. 279 51508-51515 (2004).
55. Armstrong, L. et al. A role for nucleoprotein Zap3 in the reduction of telomerase activity during embryonic stem cell differentiation. Mech. Dev. 121, 1509-1522 (2004).

56. Olnes, M. J. et al. Eltrombopag and improved hematopoiesis in refractory aplastic anemia. N. Eng. J. Med. 367, 11-19 (2012).

57. Trautmann, K. et al. Eltrombopag fails to improve severe thrombocytopenia in late-stage dyskeratosis congenita and diamond-blackfan-anaemia. Thromb. Haemost. 108, 397-398 (2012).

58. Townsley, D. M., Dumitriu, B. \& Young, N. S. Danazol treatment for telomere diseases. N. Eng. J. Med. 375, 1095-1096 (2016).

59. Calado, R. T. et al. Sex hormones, acting on the TERT gene, increase telomerase activity in human primary hematopoietic cells. Blood 114, 2236-2243 (2009).

60. Marsh, J. C. \& Mufti, G. J. Eltrombopag: a stem cell cookie? Blood 123 1774-1775 (2014).

61. Bao, W. et al. Improved regulatory T-cell activity in patients with chronic immune thrombocytopenia treated with thrombopoietic agents. Blood 116 4639-4645 (2010).

62. Martin-Ruiz, C. M., Gussekloo, J., van Heemst, D., von Zglinicki, T. \& Westendorp, R. G. J. Telomere length in white blood cells is not associated with morbidity or mortality in the oldest old: a population-based study. Aging Cell. 4, 287-290 (2005).

63. Ishaq, A., Hanson, P. S., Morris, C. M. \& Saretzki, G. Telomerase activity is downregulated early during human braindevelopment. Genes Basel. 7(6), pii: E27 (2016).

64. Andrews, S. \& FastQC, A. Quality control tool for high throughput sequence data. http://www.bioinformatics.babraham.ac.uk/projects/fastqc/.

65. Li, H. Seqtk. https://github.com//h3/seqtk.

66. $\mathrm{Xu}, \mathrm{H}$. et al. FastUniq: a fast de novo duplicates removal tool for paired short reads. PLOS. ONE. 7, e52249 (2012).

67. Li, H. \& Durbin R. Fast and accurate long-read alignment with Burrows-Wheeler transform. Bioinformatics 26, 589-595 (2010).

68. McKenna, A. et al. The genome analysis toolkit: a MapReduce framework for analyzing next-generation DNA sequencing data. Genome Res. 20, 1297-1303 (2010).

69. DePristo, M. A. et al. A framework for variation discovery and genotyping using next-generation DNA sequencing data. Nat. Genet. 43, 491-498 (2011).

70. Van der Auwera, G. A. et al. From FastQ data to high confidence variant calls: the genome analysis toolkit best practices pipeline. Curr. Protoc. Bioinforma. 43, 1-33 (2013).

71. Garrison, E. \& Marth, G. Haplotype-based variant detection from short-read sequencing (2012)

72. Genomes Project, C. et al. An integrated map of genetic variation from 1,092 human genomes. Nature 491, 56-65 (2012).

73. DuRaine, G. D., Chan, S. M. \& Reddi, A. H. Effects of TGF-beta1 on alternative splicing of superficial zone protein in articular cartilage cultures. Osteoarthr. Cartil. 19, 103-110 (2011).

74. Wang, K., Li, M. \& Hakonarson, H. ANNOVAR: functional annotation of genetic variants from high-throughput sequencing data. Nucleic Acids Res. 38, e164 (2010). 